\title{
Mikronährstoffe als Begleittherapie des kreisrunden Haarausfalls
}

\author{
Belastbare Studiendaten zum Nutzen bestimmter Spurenelemente und Vitamine bei der \\ Behandlung der Alopecia areata stehen noch aus. Einige werden allerdings seit Langem zur \\ Unterstützung der eigentlichen Therapie eingesetzt, wie der Haarexperte Dr. Andreas Finner, \\ Berlin, bestätigt.
}

Welche Behandlungsoptionen bieten Sie Ihren Patienten mit Alopecia areata an und welche Erfolgsraten sind damit zu erwarten? Finner: Je nach Stadium, Aktivität und Patientenwunsch kommen ein Abwarten beziehungsweise Kaschieren, eine äußerliche Therapie, Unterspritzungen oder Pulstherapie zum Einsatz, gelegentlich auch eine topische Immuntherapie mit Diphenylcyclopropenon, kurz DCP.

Bei neuen, kleinen, überkämmbaren Bereichen und fehlender Randaktivität im Zupftest entscheiden wir uns oft für ein Abwarten, gegebenenfalls unterstützt durch Zinkgabe und topischen Minoxidilschaum oder ein Steroid, also Clobetasolschaum. Bei stärkerer Randaktivität und längerer Bestandsdauer sowie auch für Augenbrauen kommt oft zusätzlich eine monatliche Unterspritzung mit auf 2,5 mg/ml verdünntem Triamcinolonacetonid (bis zu $6 \mathrm{ml}$ ) zum Einsatz. Dabei liegt die Erfolgsrate bei über $70 \%$. Alternativ unternehmen wir einen Therapieversuch mit PRP*.

Bei sehr aktiver, diffus-großflächiger Alopecia areata kann eine Steroidpulstherapie über fünf bis sieben Tage, angefangen mit $1 \mathrm{mg} / \mathrm{kgKG}$ täglich absteigend, sowie Pantoprazol als Magenschutz erwogen werden. Bei chronischer großflächiger Alopecia areata kann eine topische Immuntherapie mit DCP diskutiert werden, die ich jedoch wegen des hohen Aufwandes und häufiger Rezidive nach Absetzen immer seltener einsetze. Aber auch hier reicht die Erfolgsrate bis zu $70 \%$.

Es gibt Hinweise, dass bei Patienten mit Alopecia areata gehäuft ein Vitamin-D-Mangel besteht.

Die vielversprechende Anwendung von JAK-Inhibitoren ist wegen möglicher Nebenwirkungen, fehlender Zulassung und des hohen Preises noch keine etablierte Therapieoption. Wichtig ist eine einfühlsame Betreuung in der Haarsprechstunde sowie der Hinweis auf abdeckende Camouflagecremes, Haarersatz mit Zweithaar und die Selbsthilfegruppen des AAD e.V.**

Welche Rolle spielt für Sie die Supplementation von Mikronährstoffen wie Zink, Folat oder Vitamin D bei der Alopecia areata?

Finner: Die Gabe von Mikronährstoffen sehe ich bisher nur als ergänzende Möglichkeit an, insbesondere bei nachgewiesenem Mangel. Aber auch bei normalen Laborwerten rate ich zur Einnahme von Zink sowie von Kapseln mit Cystin und B-Vitaminen. Zink wird traditionell als Immunmodulator zur Begleittherapie

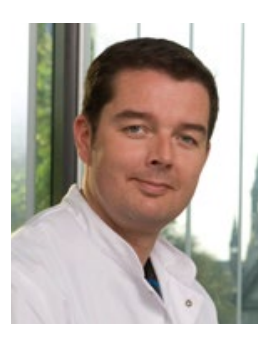

Dr. med. Andreas Finner

Praxis für Dermatologie,

Haarerkrankungen und Haartransplantation Berlin

Oft kommen die Patienten schon mit vielen Laborbefunden in die Haarsprechstunde.

empfohlen. Es gibt einige Hinweise, dass bei Patienten mit Alopecia areata gehäuft ein Vitamin-D-Mangel besteht. Die Rolle von Vitamin A, Folsäure, Selen und Vitamin $B_{12}$ ist noch unklar.

Generell denke ich aber, dass es sich eher nicht um eine direkt durch Mangelzustände ausgelöste Haarerkrankung handelt, sondern um ein genetisch vorbestimmtes Missverständnis zwischen Immunzellen und Haaren, wahrscheinlich sogar um verschiedene Mechanismen je nach Typ der Alopecia areata. Genetische Untersuchungen deuten auf Parallelen zur rheumatoiden Arthritis hin.

Bei welchen Patienten messen Sie vor der Entscheidung für eine Supplementation den Serumspiegel?

Finner: Oft kommen die Patienten schon mit vielen Laborbefunden in die Haarsprechstunde. In der Regel sind alle Laborwerte normal. Gelegentlich finden sich außerdem eine HashimotoThyreoiditis, ein Eisenmangel oder ein Vitamin-D-Mangel. Deshalb bestimme ich diese Parameter bei schweren und ausgedehnten Verlaufsformen und supplementiere bei Mangel. Bei kleinen Kahlstellen führe ich nur Laboruntersuchungen durch, wenn es zu Rezidiven kommt. Hier setzt oft schon nach zwei bis drei Monaten ein Nachwachsen ein, besonders schnell nach Unterspritzung. Zusätzliche Nahrungsergänzungen, insbesondere mit Cystin und B-Vitaminen, empfehle ich, um großflächig bereits nachwachsende Haare gut $\mathrm{zu}$ versorgen, ebenso wie bei diffusem Haarausfall oder aktiver androgenetischer Alopezie.

Das Interview führte Dr. Christine Starostzik.

*PRP = plättchenreiches Plasma

${ }^{* *}$ www.kreisrunderhaarausfall.de 